\title{
Zinc-alpha 2-glycoprotein serum level in Egyptian females with preeclampsia and eclampsia
}

\author{
Original \\ Abla A. Abou-Zeid', Eman Saad Nassar', Manal Shafik Swelem², Marian Edwar \\ Article \\ Tawfik ${ }^{1}$
}

${ }^{1}$ Department of Clinical and Chemical Pathology and ${ }^{2}$ Department of Obstetrics and Gynecology, Faculty of Medicine, Alexandria University, Egypt

\begin{abstract}
Objective: The aim of this study was to measure serum zinc- $\alpha 2$-glycoprotein (ZAG) in pregnant Egyptian females with preeclampsia and eclampsia and to correlate its levels to biochemical measures of kidney function, lipid and glucose metabolism.

Study Design: It was a retrospective study.

Patients and Methods: This study measured ZAG levels by enzyme linked immunosorbent assay (ELISA) in pregnant females with preeclampsia (PE) (no. $=40)$ and eclampsia $($ no. $=20)$ and were compared to healthy gestational age-matched subjects (no.=20). In addition, the association of ZAG with kidney function, lipid and glucose metabolism was studied. Results: Significant difference was detected on comparing the different groups regarding ZAG levels $(p=0.001)$. Furthermore, ZAG was positively correlated to systolic blood pressure, urinary protein, fasting insulin and HOMA-IR. After adjusting for other parameters, the association between ZAG and SBP, urinary protein, serum insulin and HOMA-IR remained significant by multivariate linear regression analysis.

Conclusion: The study noted that maternal ZAG serum levels are significantly increased in PE.
\end{abstract}

Key Words: Adipokines, eclampsia, preeclampsia, ZAG

Received: 02 February 2018, Accepted: 01 March 2018

Corresponding Author: Marian Tawfik, M.D., Department of Clinical Pathology, Kasr El-Aini Hospital, Cairo University, Tel.: 01221818809,E-mail: drbugsbunny05@yahoo.com

ISSN: 2090-7265, May 2018, Vol.8, No. 2

\section{INTRODUCTION}

Preeclampsia (PE) and eclampsia are serious complications that occur in pregnancy. Preeclampsia is characterized by hypertension and/or proteinuria after 20 weeks of gestation. Eclampsia is known as onset of tonic clonic seizures in a preeclamptic woman. ${ }^{[1-4]}$. As a result of a preeclamptic pregnancy, the mother and her newborn have an increased future risk for cardiovascular and metabolic diseases. $\mathrm{PE}$ and metabolic syndrome have some similar risk factors such as obesity and insulin resistance (IR). ${ }^{[2,3]}$. The pathogenesis of PE is thought to result from the imbalance between proangiogenic factors such as vascular endothelial growth factor as well as placental growth factor and anti-angiogenic factors such as soluble fms-like tyrosine kinase $1^{[2,5-8]}$. In addition, adipocytesecreted factors (adipokines) have a majorrole in the PE pathogenesis [2]. Adipose tissue is recognized as an endocrine organ producing adipokines such as adiponectin, leptin, tumour necrosis factor $\alpha$ (TNF- $\alpha$ ), chemerin and zinc- $\alpha$ 2-glycoprotein $(\mathrm{ZAG})^{[9-13]}$. Adipokines act in an autocrine/paracrine manner and/or as endocrine signals to regulateenergy expenditure, appetiteand other processes such as inflammation, angiogenesis and insulin sensitivity [13-17]. They also keep the vascular homeostasis by acting on endothelial cells. Thus, altered production of these adipokines results in the structural and functional changes in the vessels by vascular smooth muscle cell proliferation and endothelial dysfunction ${ }^{[18-19]}$. ZAG is considered as $41 \mathrm{kDa}$ soluble glycoprotein which has been found first in plasma. The name of ZAG is derived from its ability to precipitate with zinc and from its electrophoretic migration in the region of $\alpha 2-$ globulins. ${ }^{[20-22]} \mathrm{ZAG}$ is a lipid mobilizing adipokine which significantly decreases fat mass by inhibiting lipogenesis and inducing lipolysis via a cyclic AMP-mediated system and through interaction with the $\beta 3$ - adrenoreceptor ${ }^{[13,23-25]}$. It is regulated by and regulates hormones influencing glucose tolerance. It was proved that ZAG level is correlated significantly with glucose metabolism, fasting insulin, and HOMA-IR ${ }^{[22]}$. Recently, it was found that patients with hypertension have lower ZAG levels suggesting the role of ZAG in vascular homeostasis ${ }^{[18]}$. Renal clearance plays a role 
in ZAG catabolism. It was suggested that ZAG may be involved in the pathogenesis of obesity and obesity related metabolic disease; including hypertension and diabetes mellitus which represent important metabolic syndrome components ${ }^{[14-20]}$. Based on the above mentioned data, it was proposed that ZAG may have a role in the pathogenesis of PE and its complications ${ }^{[2]}$.

\section{PATIENTS AND METHODS}

Eighty pregnant females were recruited from the Obstetrics and Preeclampsia Units of Obstetrics and Gynecology Department at El-Shatby Maternity University Hospital. This study had been approved by the Ethics Committee of Alexandria Universityand a written consent was taken from each patient. Patients were divided into 3 groups; twenty pregnant females with mild preeclampsia, twenty pregnant females with severe preeclampsia, twenty pregnant females with eclampsia and twenty healthy gestational age-matched pregnant females were included as controls. PE was defined as systolic $\geq 140 \mathrm{mmHg}$ or $\geq 90 \mathrm{mmHg}$ diastolic blood pressure in combination with proteinuria in pregnant female with normal blood pressure before 20 weeks gestation. ${ }^{[4]}$ Patients with chronic hypertension, renal diseases, diabetes mellitus, endocrine diseases or chronic disease were excluded. Data for medical history, last menstrual period date, gravidity, parity and age were recorded. Blood samples were drained by venipuncture. Complete blood countwas assessed on a 3 part differential automated cell counter. Sysmex and routine chemistry investigations; fasting glucose, serum cholesterol, serum triglycerides, serum creatinine and liver function tests were performed by standard laboratory methods using Dimension RxLautoanalyzer. Estimated glomerular filtration rate (eGFR) was calculated by the Modification of Diet in Renal Disease (MDRD) study equation. ${ }^{[26]}$ Serum for ELISA assays (ZAG and insulin) was aliquoted and stored frozen at $-20^{\circ} \mathrm{C}$. Fasting insulin was determined by a commercially available ELISA (EIA-2935, DRG International, USA). Insulin sensitivity was assessed by homeostasis model assessment of insulin resistance (HOMA-IR). ${ }^{[27]} \mathrm{ZAG}$ was determined by a commercially available ELISA (BMS2201, eBioscience, Austria).

\section{STATISTICAL ANALYSIS}

Data were fed to the computer and analyzed using IBM SPSS software package version 20.0. (Armonk, NY: IBM Corp). Qualitative data were described using number and percentage. The Kolmogorov-Smirnov test was performed to verify the normality of distribution. Quantitative data were described using range (minimum and maximum), mean, standard deviation and median. Significance of the obtained results was judged at the 5\% level. Spearman coefficient was used to correlate between two distributed abnormally quantitative variables.

\section{RESULTS}

There was statistically significant difference between thestudied groups and the normal pregnant females as regards LMP, systolic and diastolic blood pressure, platelets count, protein creatinine ratio, serum creatinine, liver function tests, triglycerides levels, fasting insulin levels, fasting serum glucose and HOMAIR. Nosignificant difference was detected between the studied groups regarding age, parity, gravidity and cholesterol levels. Tables $(1-8)$.

Table 1 : Comparison between the different studied groups according to descriptive data

\begin{tabular}{|c|c|c|c|c|c|c|}
\hline \multirow[t]{2}{*}{ Obstetrics data } & $\begin{array}{l}\text { Control } \\
(\mathrm{n}=20)\end{array}$ & $\begin{array}{l}\text { Mild PE } \\
(\mathrm{n}=20)\end{array}$ & $\begin{array}{c}\text { Severe PE } \\
(\mathrm{n}=20)\end{array}$ & $\begin{array}{c}\text { Eclampsia } \\
(\mathrm{n}=20)\end{array}$ & \multirow[t]{2}{*}{ Test of Sig. } & \multirow[t]{2}{*}{$\mathrm{p}$} \\
\hline & No. & No. & No. & No. & & \\
\hline $\begin{array}{l}\text { Age (years) } \\
\text { Min. - Max. } \\
\text { Mean } \pm \text { SD. } \\
\text { Mean } \pm \text { SD. }\end{array}$ & $\begin{array}{c}19.0-42.0 \\
28.65 \pm 5.0 \\
28.50\end{array}$ & $\begin{array}{c}23.0-38.0 \\
28.55 \pm 3.87 \\
27.50\end{array}$ & $\begin{array}{c}16.0-40.0 \\
29.80 \pm 5.85 \\
30.0\end{array}$ & $\begin{array}{c}19.0-37.0 \\
31.0 \pm 5.08 \\
33.0\end{array}$ & 1.057 & 0.372 \\
\hline $\begin{array}{l}\text { LMP (weeks) } \\
\text { Min. - Max. } \\
\text { Mean } \pm \text { SD. } \\
\text { Median }\end{array}$ & $\begin{array}{c}28.50 \\
36.20 \pm 3.64 \\
38.0\end{array}$ & $\begin{array}{c}29.0-37.0 \\
33.10 \pm 2.17 \\
33.50\end{array}$ & $\begin{array}{c}27.0-36.0 \\
31.80 \pm 2.35 \\
32.50\end{array}$ & $\begin{array}{c}27.0-35.0 \\
30.85 \pm 2.11 \\
31.0\end{array}$ & $\mathrm{~F}=15.572 *$ & $<0.001 *$ \\
\hline pControl & & $0.002 *$ & $<0.001^{*}$ & $<0.001 *$ & & \\
\hline
\end{tabular}




\begin{tabular}{|c|c|c|c|c|c|c|c|c|c|c|}
\hline $\begin{array}{l}\text { Parity } \\
\text { Nulliparous } \\
\text { Multiparous }\end{array}$ & $\begin{array}{c}5 \\
15\end{array}$ & $\begin{array}{l}25.0 \\
75.0\end{array}$ & $\begin{array}{c}7 \\
13\end{array}$ & $\begin{array}{l}35.0 \\
65.0\end{array}$ & $\begin{array}{c}11 \\
9\end{array}$ & $\begin{array}{l}55.0 \\
45.0\end{array}$ & $\begin{array}{c}7 \\
13\end{array}$ & $\begin{array}{l}35.0 \\
65.0\end{array}$ & $\chi^{2}=4.053$ & 0.256 \\
\hline $\begin{array}{l}\text { Gravidity } \\
\text { Primigravida } \\
\text { Multigravida }\end{array}$ & $\begin{array}{c}3 \\
17\end{array}$ & $\begin{array}{l}15.0 \\
85.0\end{array}$ & $\begin{array}{c}7 \\
13\end{array}$ & $\begin{array}{l}35.0 \\
65.0\end{array}$ & $\begin{array}{c}11 \\
9\end{array}$ & $\begin{array}{l}55.0 \\
45.0\end{array}$ & $\begin{array}{c}7 \\
13\end{array}$ & $\begin{array}{l}35.0 \\
65.0\end{array}$ & $\chi^{2}=7.033$ & 0.071 \\
\hline $\begin{array}{l}\text { Min. - Max. } \\
\text { Median }\end{array}$ & \multicolumn{2}{|c|}{$\begin{array}{c}1.0-6.0 \\
2.50\end{array}$} & \multicolumn{2}{|c|}{$\begin{array}{c}1.0-5.0 \\
2.0\end{array}$} & \multicolumn{2}{|c|}{$\begin{array}{c}1.0-6.0 \\
1.0\end{array}$} & \multicolumn{2}{|c|}{$\begin{array}{c}1.0-5.0 \\
2.0\end{array}$} & $\mathrm{H}=6.014$ & 0.111 \\
\hline
\end{tabular}

$\chi^{2}, \mathrm{p}: \chi^{2}$ and $\mathrm{p}$ values for Chi square test for comparing between the different groups

F,p: F and p values for ANOVA test, Sig. bet. grps was done using Post Hoc Test (Tukey)

$\mathrm{H}, \mathrm{p}$ : $\mathrm{H}$ and $\mathrm{p}$ values for Kruskal Wallis test

pControl: $p$ value for comparing between control and each other group

$\mathrm{p} 1$ : $\mathrm{p}$ value for comparing between mild and severe

$\mathrm{p} 2$ : $\mathrm{p}$ value for comparing between mild and eclampsia

p3: $\mathrm{p}$ value for comparing between severe and eclampsia

*: Statistically significant at $p \leq 0.05$

Table 2: Comparison between the different studied groups according to blood pressure

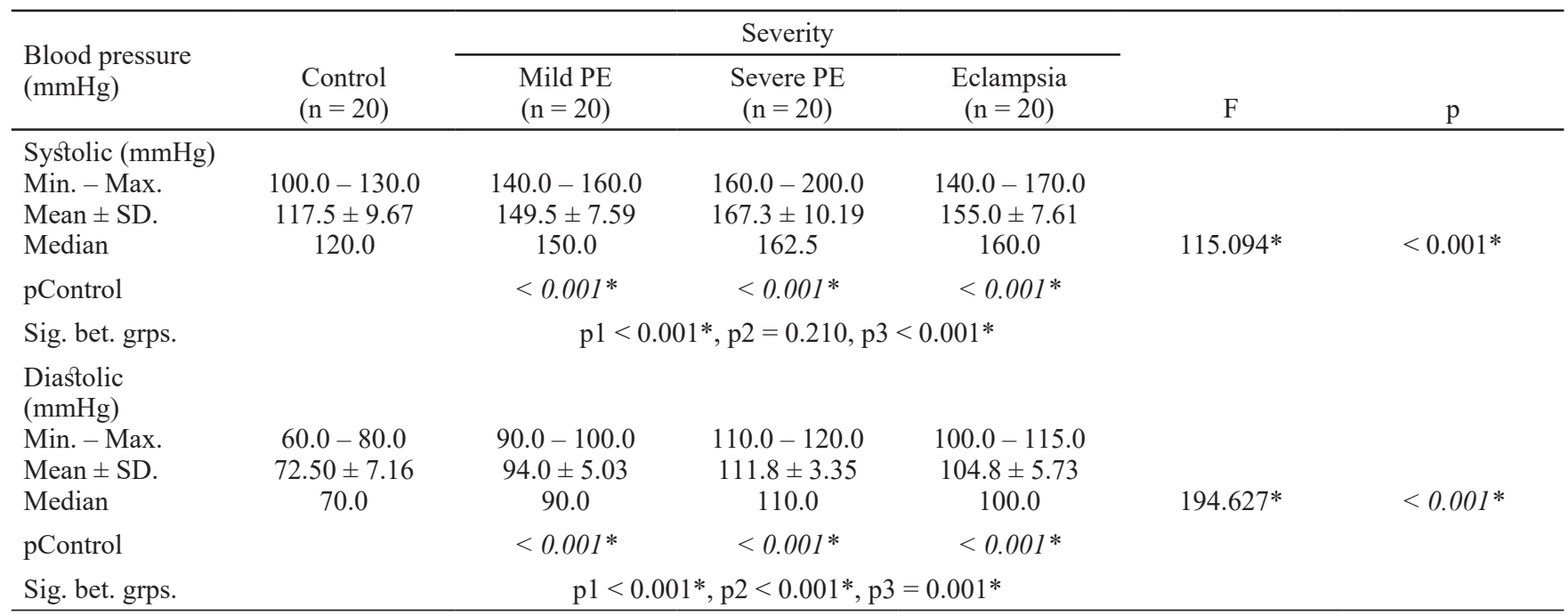

Table 3 : Comparison between the different studied groups according to $\mathrm{CBC}$

\begin{tabular}{|c|c|c|c|c|c|c|}
\hline & \multirow[b]{2}{*}{$\begin{array}{l}\text { Control } \\
(\mathrm{n}=20)\end{array}$} & \multicolumn{3}{|c|}{ Severity } & \multirow[b]{2}{*}{$\mathrm{F}$} & \multirow[b]{2}{*}{$\mathrm{p}$} \\
\hline & & $\begin{array}{l}\text { Mild PE } \\
(\mathrm{n}=20)\end{array}$ & $\begin{array}{c}\text { Severe PE } \\
(\mathrm{n}=20)\end{array}$ & $\begin{array}{c}\text { Eclampsia } \\
(\mathrm{n}=20)\end{array}$ & & \\
\hline $\begin{array}{l}\text { Haemoglobin } \\
(\mathrm{g} / \mathrm{dl}) \\
\text { Min. - Max. } \\
\text { Mean } \pm \text { SD. } \\
\text { Median }\end{array}$ & $\begin{array}{c}9.0-12.0 \\
10.13 \pm 1.06 \\
10.0\end{array}$ & $\begin{array}{l}8.40-11.0 \\
9.73 \pm 0.84 \\
9.95\end{array}$ & $\begin{array}{c}8.50-11.0 \\
9.75 \pm 0.70 \\
9.80\end{array}$ & $\begin{array}{c}8.80-11.0 \\
9.65 \pm 0.69 \\
9.60\end{array}$ & 1.293 & 0.283 \\
\hline $\begin{array}{l}\text { WBCs }(/ \mu 1) \\
\text { Min. - Max. } \\
\text { Mean } \pm \text { SD. } \\
\text { Median }\end{array}$ & $\begin{array}{c}4000-11000 \\
7520 \pm 2169.79 \\
8000\end{array}$ & $\begin{array}{c}4300-11000 \\
7440 \pm 1919.54 \\
7500\end{array}$ & $\begin{array}{c}5300-11500 \\
7715 \pm 1912.36 \\
7800\end{array}$ & $\begin{array}{c}4000-12100 \\
7265 \pm 2446.54 \\
7050\end{array}$ & 0.155 & 0.926 \\
\hline $\begin{array}{l}\text { Platelets } \\
(\times 103 / \mu l) \\
\text { Min. }- \text { Max. } \\
\text { Mean } \pm \text { SD. } \\
\text { Median }\end{array}$ & $\begin{array}{c}155.0-320.0 \\
203.8 \pm 43.70 \\
188.5\end{array}$ & $\begin{array}{c}120.0-250.0 \\
173.8 \pm 32.75 \\
170.5\end{array}$ & $\begin{array}{c}90.0-127.0 \\
107.4 \pm 9.89 \\
104.5\end{array}$ & $\begin{array}{c}92.0-138.0 \\
113.2 \pm 12.77 \\
111.0\end{array}$ & $54.463 *$ & $<0.001^{*}$ \\
\hline pControl & & $0.007^{*}$ & $<0.001^{*}$ & $<0.001^{*}$ & & \\
\hline Sig. bet. grps. & \multicolumn{5}{|c|}{$\mathrm{p} 1<0.001 *, \mathrm{p} 2<0.001^{*}, \mathrm{p} 3=0.919$} & \\
\hline
\end{tabular}


Table 4 : Comparison between the different studied groups according to urinary protein, urinary creatinine and PCR

\begin{tabular}{|c|c|c|c|c|c|c|}
\hline & \multirow{2}{*}{$\begin{array}{l}\text { Control } \\
(\mathrm{n}=20)\end{array}$} & \multicolumn{3}{|c|}{ Severity } & \multirow[b]{2}{*}{$\mathrm{H}$} & \multirow[b]{2}{*}{$\mathrm{p}$} \\
\hline & & $\begin{array}{l}\text { Mild PE } \\
(\mathrm{n}=20)\end{array}$ & $\begin{array}{l}\text { Severe PE } \\
(\mathrm{n}=20)\end{array}$ & $\begin{array}{c}\text { Eclampsia } \\
(\mathrm{n}=20)\end{array}$ & & \\
\hline $\begin{array}{l}\text { Urinary protein } \\
(\mathrm{mg} / \mathrm{dl}) \\
\text { Min. }- \text { Max. } \\
\text { Mean } \pm \text { SD. } \\
\text { Median }\end{array}$ & $\begin{array}{c}2.0-23.0 \\
8.71 \pm 8.11 \\
4.0\end{array}$ & $\begin{array}{c}10.0-230.0 \\
54.25 \pm 47.96 \\
37.50\end{array}$ & $\begin{array}{c}55.0-340.0 \\
178.3 \pm 90.78 \\
173.0\end{array}$ & $\begin{array}{c}11.0-831.0 \\
143.6 \pm 180.1 \\
94.0\end{array}$ & $55.033^{*}$ & $<0.001^{*}$ \\
\hline pControl & & $<0.001 *$ & $<0.001 *$ & $<0.001 *$ & & \\
\hline Sig. bet. grps. & \multicolumn{5}{|c|}{$\mathrm{p} 1<0.001 *, \mathrm{p} 2=0.002 *, \mathrm{p} 3=0.030 *$} & \\
\hline $\begin{array}{l}\text { Urinary } \\
\text { creatinine } \\
(\mathrm{mg} / \mathrm{dl}) \\
\text { Min. - Max. } \\
\text { Mean } \pm \text { SD. } \\
\text { Median }\end{array}$ & $\begin{array}{c}42.81-334.1 \\
107.7 \pm 63.47 \\
96.56\end{array}$ & $\begin{array}{c}24.02-167.3 \\
87.81 \pm 38.63 \\
70.97\end{array}$ & $\begin{array}{c}11.0-69.53 \\
33.36 \pm 15.24 \\
33.10\end{array}$ & $\begin{array}{c}12.46-136.5 \\
29.99 \pm 29.40 \\
18.01\end{array}$ & $45.442 *$ & $<0.001^{*}$ \\
\hline pControl & & 0.372 & $<0.001 *$ & $<0.001^{*}$ & & \multirow{5}{*}{$<0.001^{*}$} \\
\hline Sig. bet. grps. & \multicolumn{5}{|c|}{$p 1<0.001^{*}, p 2<0.001^{*}, p 3=0.074$} & \\
\hline $\begin{array}{l}\text { PCR } \\
(\mathrm{mg} / \mathrm{g}) \\
\text { Min. - Max. } \\
\text { Mean } \pm \text { SD. } \\
\text { Median }\end{array}$ & $\begin{array}{c}11.30-169.0 \\
79.07 \pm 51.85 \\
68.05\end{array}$ & $\begin{array}{c}314.3-1892.2 \\
622.7 \pm 419.5 \\
414.3\end{array}$ & $\begin{array}{c}2038.0-10757.0 \\
6065.6 \pm 2879.3 \\
5399.6\end{array}$ & $\begin{array}{c}881.4-7275.0 \\
4095.3 \pm 1795.7 \\
3719.8\end{array}$ & $67.047^{*}$ & \\
\hline pControl & & $<0.001 *$ & $<0.001 *$ & $<0.001^{*}$ & & \\
\hline Sig. bet. grps. & & $p 1<0$. & $1 *, p 2<0.001^{*}, p$ & $=0.051$ & & \\
\hline
\end{tabular}

Table 5 : Comparison between the different studied groups according to serum creatinine and eGFR

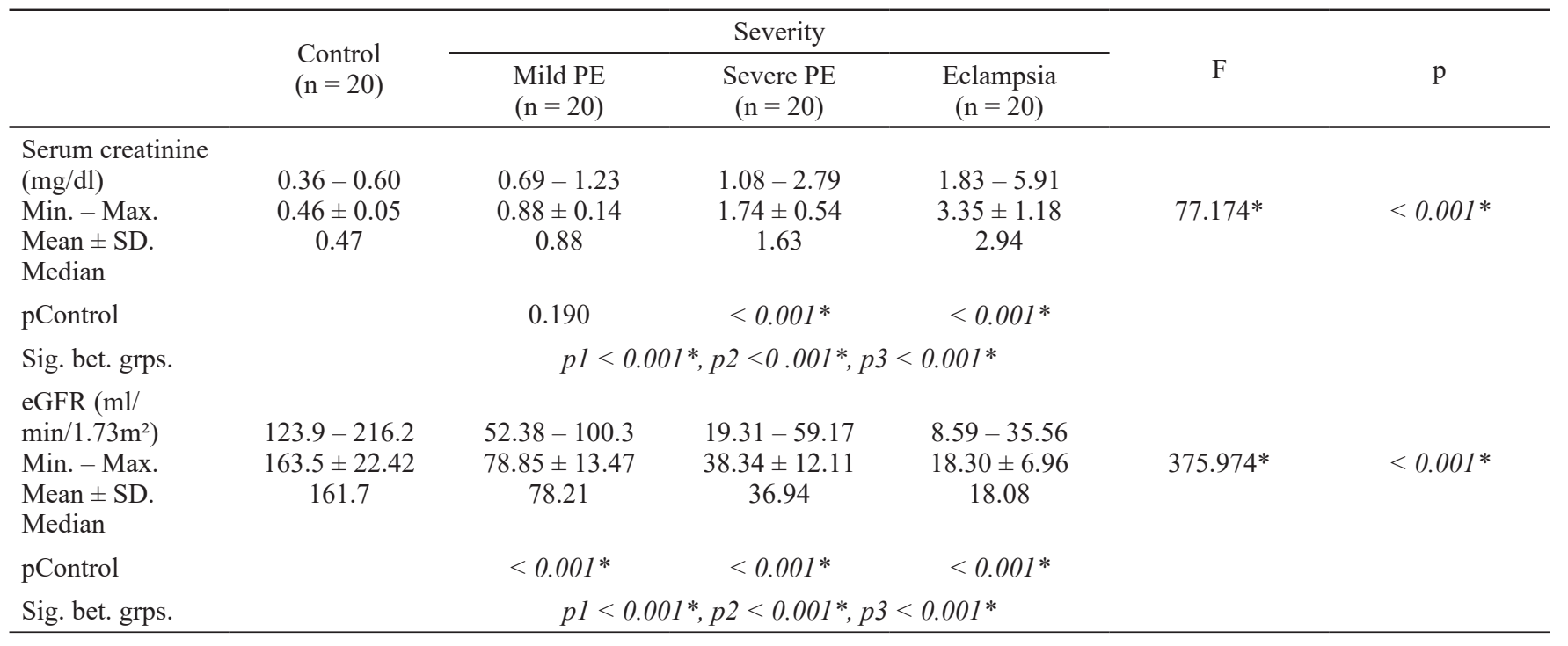


Table 6 : Comparison between the different studied groups according to lipid profile

\begin{tabular}{|c|c|c|c|c|c|c|}
\hline \multirow[b]{2}{*}{ Lipid profile } & \multirow[b]{2}{*}{$\begin{array}{l}\text { Control } \\
(\mathrm{n}=20)\end{array}$} & \multicolumn{3}{|c|}{ Severity } & \multirow[b]{2}{*}{ Test of Sig. } & \multirow[b]{2}{*}{$\mathrm{p}$} \\
\hline & & $\begin{array}{l}\text { Mild PE } \\
(\mathrm{n}=20)\end{array}$ & $\begin{array}{l}\text { Severe PE } \\
(\mathrm{n}=20)\end{array}$ & $\begin{array}{l}\text { Eclampsia } \\
(\mathrm{n}=20)\end{array}$ & & \\
\hline \multicolumn{7}{|l|}{ Triglycerides (mg/dl) } \\
\hline Min. - Max. & $50.0-245.0$ & $114.0-400.0$ & $100.0-385.0$ & $137.0-343.0$ & $\mathrm{H}=$ & \multirow{4}{*}{$0.001 *$} \\
\hline Mean \pm SD. & $159.45 \pm 43.29$ & $218.10 \pm 91.66$ & $235.70 \pm 65.38$ & $226.35 \pm 60.10$ & $16.707 *$ & \\
\hline Median & 169.50 & 179.0 & 217.0 & 206.50 & & \\
\hline pControl & & 0.104 & $<0.001^{*}$ & $0.001 *$ & & \\
\hline Sig. bet. grps. & \multicolumn{5}{|c|}{$\mathrm{p} 1=0.234, \mathrm{p} 2=0.402, \mathrm{p} 3=0.588$} & \\
\hline Cholesterol (mg/dl) & & & & & & \multirow{4}{*}{0.524} \\
\hline Min. - Max. & $80.0-278.0$ & $140.0-292.0$ & $123.0-291.0$ & $150.0-287.0$ & $\mathrm{~F}=$ & \\
\hline Mean \pm SD. & $211.75 \pm 42.75$ & $211.70 \pm 40.63$ & $217.35 \pm 50.55$ & $197.15 \pm 43.54$ & 0.754 & \\
\hline Median & 217.50 & 217.50 & 224.0 & 179.50 & & \\
\hline
\end{tabular}

Table 7 : Comparison between the different studied groups according to liver function (ALT, AST)

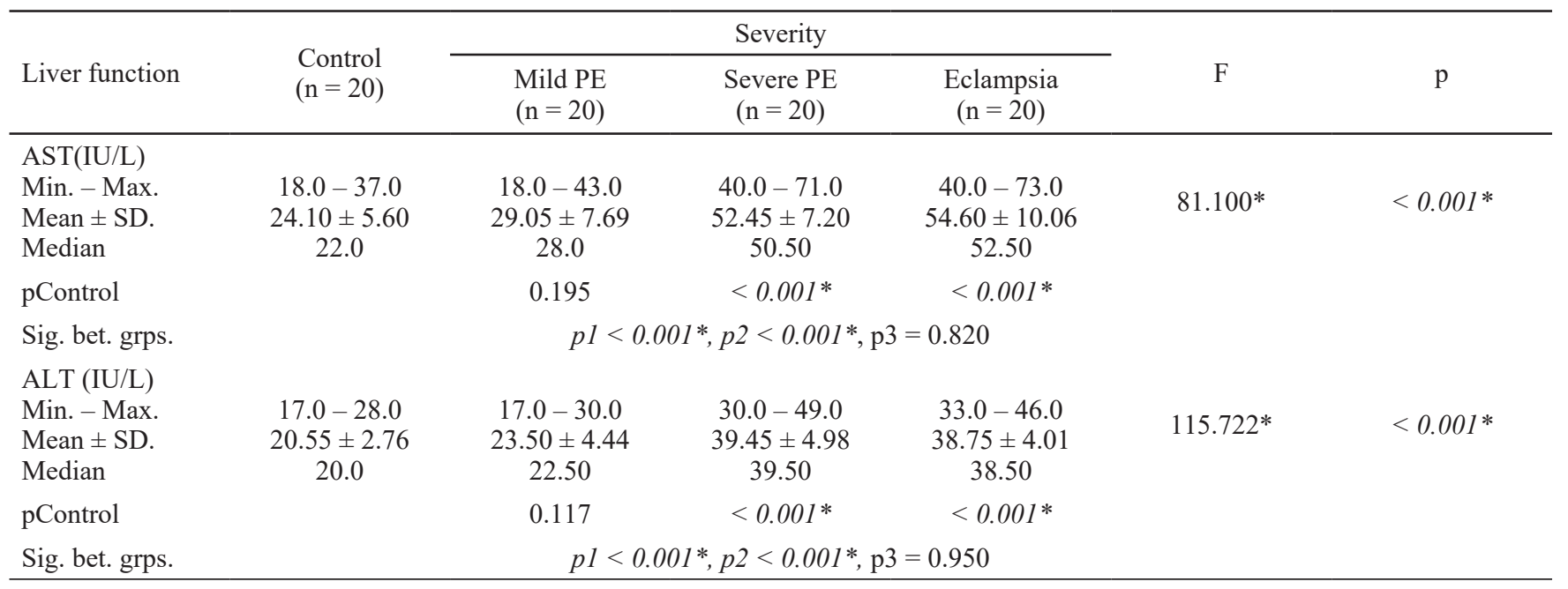

Table 8 : Comparison between the different studied groups according to fasting serum glucose, fasting serum insulin and HOMA-IR

\begin{tabular}{|c|c|c|c|c|c|c|}
\hline & $\begin{array}{l}\text { Control } \\
(\mathrm{n}=20)\end{array}$ & $\begin{array}{l}\text { Mild PE } \\
(\mathrm{n}=20)\end{array}$ & $\begin{array}{l}\text { Severe PE } \\
(\mathrm{n}=20)\end{array}$ & $\begin{array}{l}\text { Eclampsia } \\
(\mathrm{n}=20)\end{array}$ & Test of Sig. & $\mathrm{p}$ \\
\hline $\begin{array}{l}\text { Fasting serum glucose }(\mathrm{mg} / \mathrm{dl}) \\
\text { Min. - Max. } \\
\text { Mean } \pm \text { SD. } \\
\text { Median }\end{array}$ & $\begin{array}{l}60.0-89.0 \\
72.20 \pm 7.92 \\
71.0\end{array}$ & $\begin{array}{l}68.0-147.0 \\
103.2 \pm 21.69 \\
95.50\end{array}$ & $\begin{array}{l}78.0-185.0 \\
125.1 \pm 34.45 \\
117.0\end{array}$ & $\begin{array}{l}98.0-181.0 \\
134.9 \pm 19.72 \\
132.0\end{array}$ & $\begin{array}{l}\mathrm{F}= \\
29.301 *\end{array}$ & $<0.001 *$ \\
\hline pControl & & $<0.001^{*}$ & $<0.001 *$ & $<0.001^{*}$ & & \\
\hline Sig. bet. grps. & & \multicolumn{3}{|c|}{$\mathrm{p} 1=0.018^{*}, \mathrm{p} 2<0.001^{*}, \mathrm{p} 3=0.530$} & & \\
\hline $\begin{array}{l}\text { Fasting serum insulin }(\mu \mathrm{IU} / \mathrm{mL}) \\
\text { Min. - Max. } \\
\text { Mean } \pm \text { SD. } \\
\text { Median }\end{array}$ & $\begin{array}{l}6.20-34.07 \\
13.69 \pm 8.56 \\
11.14\end{array}$ & $\begin{array}{l}6.20-45.40 \\
24.15 \pm 13.20 \\
23.79\end{array}$ & $\begin{array}{l}8.19-97.35 \\
36.73 \pm 28.17 \\
24.79\end{array}$ & $\begin{array}{l}6.30-54.09 \\
10.69 \pm 10.45 \\
7.55\end{array}$ & \multirow[t]{2}{*}{$\begin{array}{l}H= \\
26.666^{*}\end{array}$} & \multirow[t]{2}{*}{$<0.001^{*}$} \\
\hline pControl & & $0.007^{*}$ & $0.001 *$ & 0.198 & & \\
\hline Sig. bet. grps. & & \multicolumn{3}{|c|}{$\mathrm{p} 1=0.234, \mathrm{p} 2=0.003^{*}, \mathrm{p} 3<0.001^{*}$} & \multirow{4}{*}{$\begin{array}{l}\mathrm{H}= \\
32.494 *\end{array}$} & \multirow{4}{*}{$<0.001 *$} \\
\hline $\begin{array}{l}\text { HOMA-IR } \\
\text { Min. - Max. } \\
\text { Mean } \pm \text { SD. } \\
\text { Median }\end{array}$ & $\begin{array}{l}0.96-5.46 \\
2.31 \pm 1.27 \\
2.15\end{array}$ & $\begin{array}{l}1.09-13.40 \\
6.17 \pm 3.83 \\
5.45\end{array}$ & $\begin{array}{l}2.50-38.30 \\
12.81 \pm 12.17 \\
7.05\end{array}$ & $\begin{array}{l}2.30-16.43 \\
3.42 \pm 3.11 \\
2.51\end{array}$ & & \\
\hline pControl & & $<0.001^{*}$ & $<0.001 *$ & $0.026^{*}$ & & \\
\hline Sig. bet. grps. & & \multicolumn{3}{|c|}{$\mathrm{p} 1=0.133, \mathrm{p} 2=0.010^{*}, p 3<0.001^{*}$} & & \\
\hline
\end{tabular}


Statistically significant difference was detected on comparing the different groups regarding ZAG levels in the serum $(p=0.001)$. Table 9 and Figure 1. A positive correlation was found between ZAG and systolic blood pressure $(\mathrm{r}=0.305, p=0.018)$, urinary protein $(\mathrm{r}=$ $0.316, p=0.014)$, fasting insulin $(\mathrm{r}=0.303, p=0.019)$ and HOMA-IR $(\mathrm{r}=0.261, p=0.044)$ in the patients group by univariate correlation. Table (11). In the patients group, the association between ZAG and SBP $(\beta=0.354$, $p=0.013)$, urinary protein $(\beta=0.310, p=0.014)$, serum insulin $(\beta=1.259, \quad \mathrm{p}=0.005)$ and HOMA-IR $(\beta=-1.189, p=0.013)$ remained significant after adjusting for other parameters by multivariate linear regression analysis. Table (11).

Regarding the performance of ZAG for diagnosing preeclampsia/eclampsia, ROC curve analysis showed that at a cut off $>146.1 \mu \mathrm{g} / \mathrm{ml}$, ZAG had a diagnostic sensitivity of $75.0 \%$ and a specificity of $60.0 \%$. Figure (2), Table (12)

Table 9 : Comparison between the different studied groups according to ZAG

\begin{tabular}{|c|c|c|c|c|c|c|}
\hline & $\begin{array}{l}\text { Control } \\
(n=20)\end{array}$ & $\begin{array}{l}\text { Mild PE } \\
(n=20)\end{array}$ & $\begin{array}{c}\text { Severe PE } \\
(\mathrm{n}=20)\end{array}$ & $\begin{array}{c}\text { Eclampsia } \\
(n=20)\end{array}$ & $\mathrm{F}$ & $\mathrm{p}$ \\
\hline \multicolumn{7}{|l|}{$\mathrm{ZAG}(\mu \mathrm{g} / \mathrm{ml})$} \\
\hline Min. - Max. & $75.9-160.4$ & $87.8-157.6$ & $133.3-166.8$ & $59.2-157.6$ & \multirow{3}{*}{$6.065^{*}$} & \multirow{3}{*}{$0.001 *$} \\
\hline Mean \pm SD & $126.7 \pm 31.6$ & $146.5 \pm 14.1$ & $151.3 \pm 7.3$ & $126.7 \pm 31.1$ & & \\
\hline Median & 144.6 & 148.3 & 149.9 & 142.3 & & \\
\hline pControl & & $0.047 *$ & $0.008 *$ & 1.000 & & \\
\hline Sig. bet. grps. & \multicolumn{5}{|c|}{$\mathrm{p} 1=0.916, \mathrm{p} 2=0.046^{*}, \mathrm{p} 3=0.008^{*}$} & \\
\hline
\end{tabular}

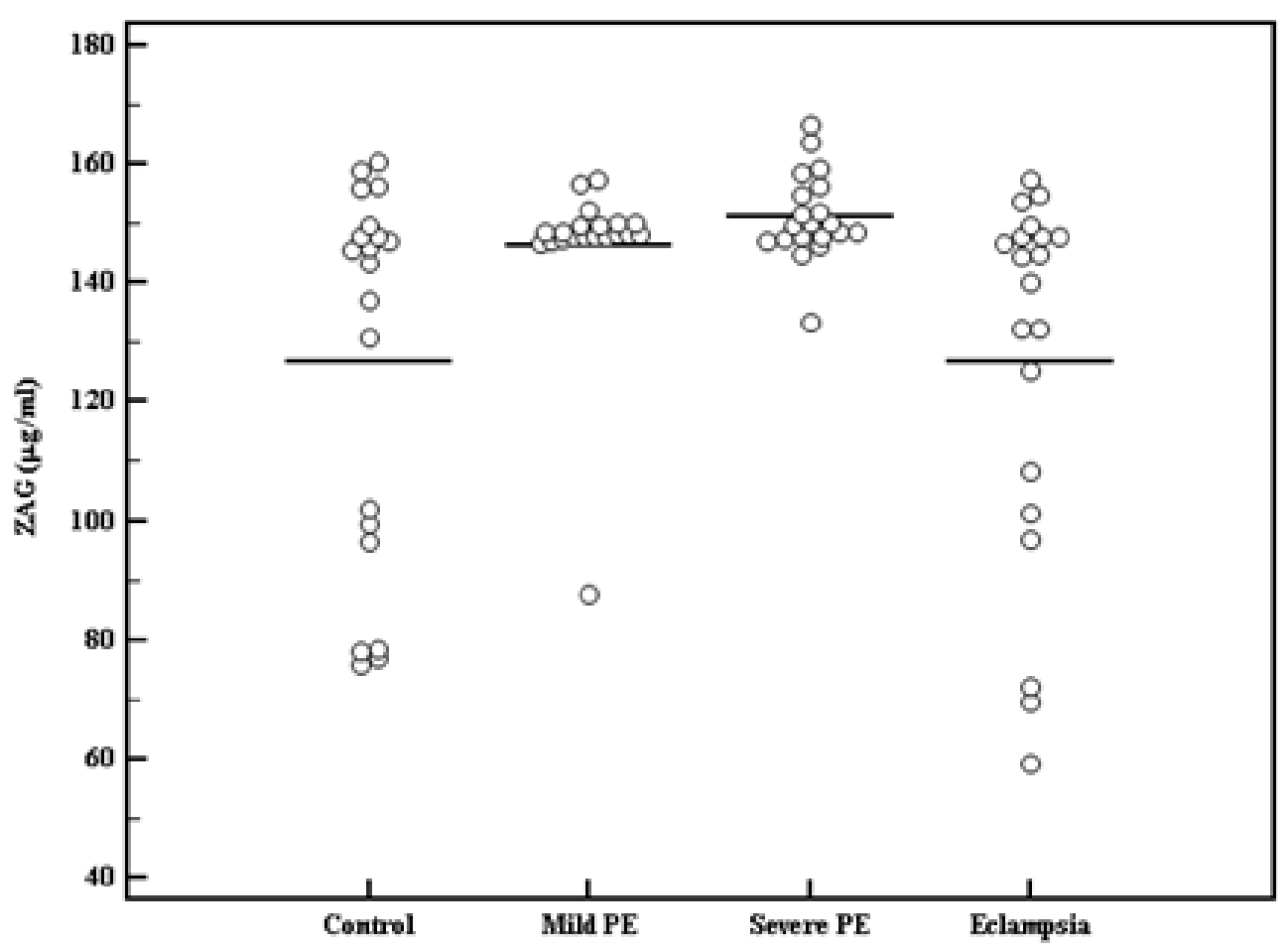

Fig. 1 : Comparison between the different studied groups according to ZAG 
Table 10 : Univariate correlation between ZAG $(\mu \mathrm{g} / \mathrm{ml})$ and different parameters

\begin{tabular}{|c|c|c|c|c|c|c|}
\hline & \multicolumn{6}{|c|}{$\mathrm{ZAG}(\mu \mathrm{g} / \mathrm{ml})$} \\
\hline & \multicolumn{2}{|c|}{$\begin{array}{l}\text { Total sample } \\
\quad(\mathrm{n}=80)\end{array}$} & \multicolumn{2}{|c|}{$\begin{array}{l}\text { Patients } \\
(\mathrm{n}=60)\end{array}$} & \multicolumn{2}{|c|}{$\begin{array}{l}\text { Control } \\
(\mathrm{n}=20)\end{array}$} \\
\hline & $\mathrm{r}$ & $\mathrm{p}$ & $\mathrm{r}$ & $\mathrm{p}$ & $\mathrm{r}$ & $\mathrm{p}$ \\
\hline Age (years) & 0.109 & 0.334 & 0.106 & 0.421 & 0.052 & 0.828 \\
\hline LMP (weeks) & -0.046 & 0.683 & 0.122 & 0.353 & 0.117 & 0.623 \\
\hline Gravidity & -0.010 & 0.929 & 0.074 & 0.573 & -0.046 & 0.848 \\
\hline Systolic blood pressure (mmHg) & $0.291 *$ & $0.009 *$ & $0.305^{*}$ & $0.018^{*}$ & -0.226 & 0.337 \\
\hline Diastolic blood pressure $(\mathrm{mmHg})$ & 0.195 & 0.083 & 0.021 & 0.874 & -0.193 & 0.415 \\
\hline Urinary protein $(\mathrm{mg} / \mathrm{dl})$ & $0.332 *$ & $0.003^{*}$ & $0.316^{*}$ & $0.014^{*}$ & 0.533 & $0.016^{*}$ \\
\hline Urinary creatinine $(\mathrm{mg} / \mathrm{dl})$ & -0.099 & 0.382 & -0.088 & 0.501 & 0.178 & 0.453 \\
\hline Serum Creatinine $(\mathrm{mg} / \mathrm{dl})$ & 0.008 & 0.941 & -0.185 & 0.156 & -0.104 & 0.662 \\
\hline eGFR $\left(\mathrm{ml} / \mathrm{min} / 1.73 \mathrm{~m}^{2}\right)$ & -0.145 & 0.200 & 0.235 & 0.071 & 0.041 & 0.863 \\
\hline Triglycerides (mg/dl) & 0.153 & 0.177 & 0.147 & 0.263 & -0.256 & 0.276 \\
\hline Total cholesterol (mg/dl) & 0.011 & 0.921 & 0.087 & 0.507 & -0.139 & 0.560 \\
\hline Fasting serum glucose $(\mathrm{mg} / \mathrm{dl})$ & 0.101 & 0.372 & -0.072 & 0.586 & -0.267 & 0.254 \\
\hline Fasting serum insulin $(\mu \mathrm{IU} / \mathrm{mL})$ & $0.286^{*}$ & $0.010^{*}$ & $0.303 *$ & $0.019^{*}$ & 0.084 & 0.724 \\
\hline HOMA-IR & $0.264 *$ & $0.018 *$ & $0.261 *$ & $0.044^{*}$ & 0.048 & 0.841 \\
\hline
\end{tabular}

$\mathrm{r}$ : Pearson coefficient

*: Statistically significant at $p \leq 0.05$

Table 11 : Multivariate linear regression analysis for ZAG $(\mu \mathrm{g} / \mathrm{ml})$ in patients group

\begin{tabular}{llll}
\hline & Beta & t & p \\
\hline Systolic blood pressure $(\mathrm{mmHg})$ & 0.354 & $2.553^{*}$ & $0.013^{*}$ \\
Urinary protein $(\mathrm{mg} / \mathrm{dl})$ & 0.310 & $2.548^{*}$ & $0.014^{*}$ \\
Serum insulin $(\mu \mathrm{IU} / \mathrm{mL})$ & 1.259 & $2.892^{*}$ & $0.005^{*}$ \\
HOMA IR & -1.189 & $2.558^{*}$ & $0.013^{*}$ \\
\hline
\end{tabular}

Beta: Standardized Coefficients 


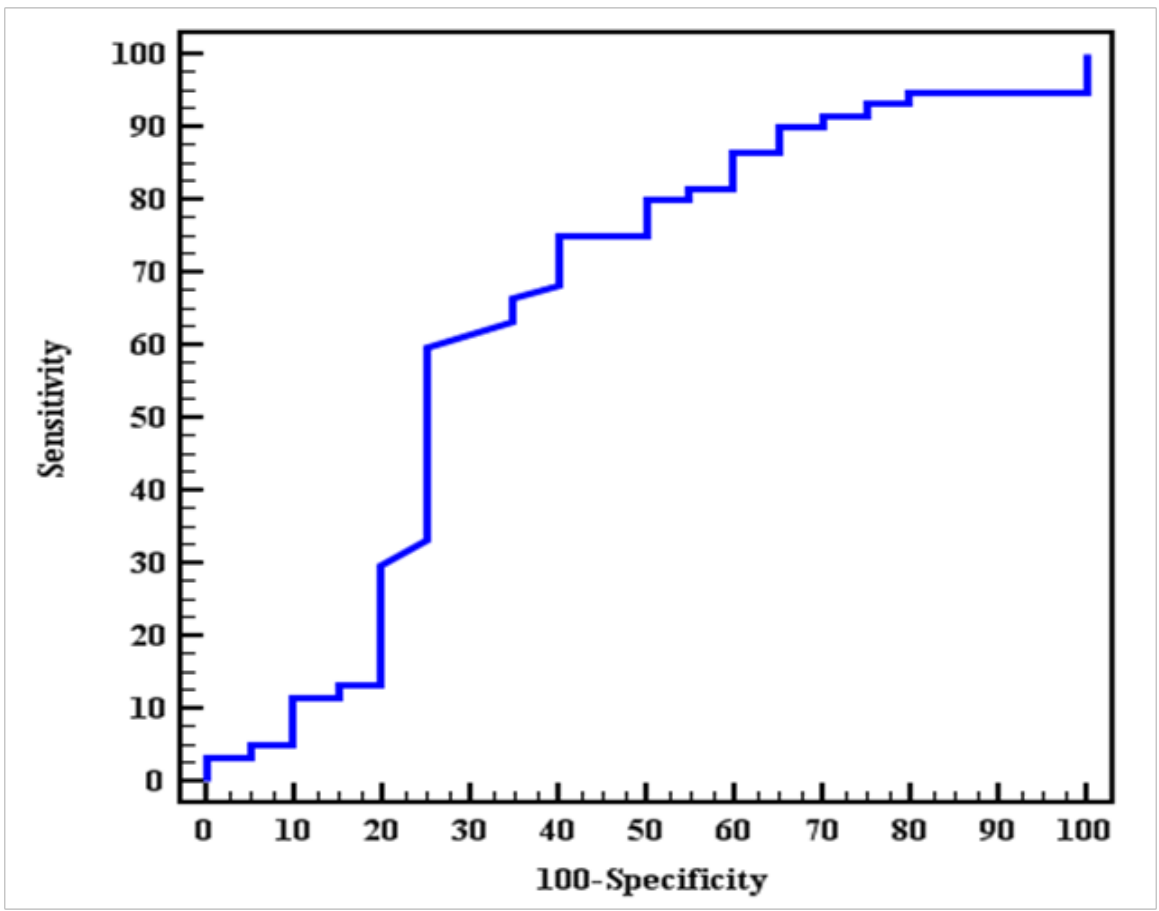

Fig. 2 : ROC curve for ZAG $(\mu \mathrm{g} / \mathrm{ml})$ to diagnose patients from control

Table 12 : Agreement (sensitivity, specificity) for ZAG $(\mu \mathrm{g} / \mathrm{ml})$ to diagnose patientsfrom control

\begin{tabular}{|c|c|c|c|c|c|c|c|c|c|}
\hline & \multirow{2}{*}{ AUC } & \multirow{2}{*}{$\mathrm{p}$} & \multicolumn{2}{|c|}{$95 \%$ C.I } & \multirow{2}{*}{ Cut off } & \multirow{2}{*}{ Sensitivity } & \multirow{2}{*}{ Specificity } & \multirow{2}{*}{ PPV } & \multirow{2}{*}{ NPV } \\
\hline & & & LL & UL & & & & & \\
\hline $\mathrm{ZAG}(\mu \mathrm{g} / \mathrm{ml})$ & $0.655^{*}$ & $0.039 *$ & 0.500 & 0.809 & $>146.1$ & 75.0 & 60.0 & 84.9 & 44.4 \\
\hline
\end{tabular}

\section{DISCUSSION}

In our study, maternal ZAG was significantly increased in mild and severe preeclampsia subjects as compared to healthy pregnant controls. The results may be attributed to that systemic endothelial dysfunction occurs in preeclampsia which affects glomerular epithelial cells and causes renal injury. Renal degradation contributes to ZAG clearance and elimination. Therefore, renal impairment may result in elevation of ZAG concentration. ${ }^{[28]}$

In agreement with these findings, Stepan $\mathrm{H}$ et al. found that the median ZAG was 1.4-fold higher in preeclamptic patients when compared to controls. This is in accordance with the hypothesis that adipokines such as ZAG may have a role in preeclampsia pathogenesis and its complications. ${ }^{[2]}$

Furthermore, the current study found a positive correlation between circulating ZAG on one hand and SBP, urinary protein, fasting insulin and HOMA-IR on the other hand in the patients group. The association remained significant after adjusting for other parameters that may affect levels of ZAG in patients.

However, Stepan H. and his colleagues ${ }^{[2]}$ reported that ZAG was positively associated only with SBP, DBP, creatinine and TG. No correlation between circulating ZAG and fasting glucose, fasting insulin as well as HOMA-IR and age was found. Only serum creatinine remained a strong independent predictor of ZAG concentrations in multiple regression analysis. These results had pointed out that ZAG depended on kidney function and that renal elimination was a major route by which physiologic ZAG serum levels are maintained.

The results were supported by other researchers, who suggested that increased ZAG concentration in CKD patients and chronic hemodialysis patients could be attributed to decreased GFR and/or tubular reabsorption which in turn decreases the renal degradation of ZAG. ${ }^{[28-30]}$

Also, Leal $\mathrm{V}$ et al. [31] found that ZAG was significantly increased in regular hemodialysis patients 
when compared to controls. In addition, markers of inflammation, interleukin-6 and CRP were increased in patients on hemodialysis. It has been proposed that ZAG is an adipokine with anti-inflammatory properties. Thus, elevation of ZAG levels might be linked to oxidative stress and inflammation occurred in renal patients on hemodialysis and might reflect resolution of the pro-inflammatory process. ${ }^{[32]}$

In contrast with this study, Zhu $\mathrm{H}$ et al. ${ }^{[18]}$ reported lower ZAG levels in hypertensive subjects with normal kidney function compared to controls. The median ZAG levels were $21.6 \%$ lower in hypertensive subjects. They concluded that ZAG may have a role in blood pressure control and that the lower ZAG levels in hypertensive subjects was related to blood pressure but not to renal function. The different change trend of ZAG concentrations in preeclampsia and hypertensive patients may be explained by the different pathogenesis of these two diseases.

Yang $\mathrm{M}$ et al. ${ }^{[22]}$ reported significantly lower levels of ZAG in newly diagnosed diabetes mellitus (DM) patients or impaired glucose tolerance (IGT) compared with controls with normal glucose tolerance. HOMAIR was independently related with serum ZAG. Also, obese or overweight individuals had significantly decreased ZAG concentrations than lean individuals. In addition, they found that ZAG mRNA expression and ZAG protein were down-regulated in adipose tissues from newly diagnosed DM patients compared with controls. A negative correlation was noted between ZAG level and dyslipidemia, adiposity, glucose metabolism, fasting insulin as well as HOMA-IR. These findings suggested that circulating ZAG might be linked to obesity and insulin resistance through its interaction with $\beta 3$-adrenoreceptors and stimulating lipolysis suggesting a role in the regulation of lipid metabolism and insulin sensitivity. So, it could be used as a novel biomarker for insulin resistance syndrome and DM. ${ }^{[33,34]}$

However, $\mathrm{Xu} \mathrm{L}$ et al. ${ }^{[35]}$ reported that the diabetic patients with higher ZAG concentrations had decreased eGFR than those with low ZAG concentrations. This result suggested that serum $Z A G$ concentrations were increased in T2DM patients complicated by diabetic nephropathy which is a microvascular complication that leads to slow deterioration of the kidneys and finally to end-stage renal disease. ZAG was found to be negatively correlated with eGFR in diabetic patients.

Moreover, Marrades M. et al. ${ }^{[23]}$ revealed that the expression of gene of ZAG was decreased in adipose tissue of obese subjects compared to lean subjects. These results suggested that ZAG might play a major role in the regulation of adipose tissue metabolism. The down regulation of ZAG gene in obese subjects could be the cause of an impairment of lipid-mobilization, increasing the possibility that ZAG might be a candidate gene in the control of body weight and obesity related disorders.

On the other hand, Yeung D et al. ${ }^{[36]}$ found that obese individuals had significantly increased ZAG concentrations than lean individuals. Furthermore, significantly higher ZAG levels were observed in patients with dyslipidemia, hypertension and type 2 DM. In addition, ZAG correlated positively with diastolic blood pressure, fasting insulin, insulin resistance indices and parameters of adiposity. These findings suggested that the elevation of ZAG in subjects with obesity might be a compensatory upregulation to counteract the metabolic stress imposed by obesity. Also, it is possible that obesity may cause resistance to ZAG actions leading to its compensatory up regulation. The authors concluded that circulating ZAG might play a regulatory role in obesity-related metabolic syndrome and metabolism of lipid.

In contrast, Stejskal D et al. ${ }^{[37]}$ reported that ZAG level did not differentiate healthy subjects from subjects with metabolic syndrome. This might be explained by the influence of hormonal interactions, food intake or energy balance on ZAG level or might result from the fact that ZAG is secreted in various tissues and fluids of the body. These findings corroborated those of other authors who noted that the difference between obese subjects with high insulin resistance and those with low insulin resistance regarding ZAG was not significant. These results suggested that ZAG might be closely linked mainly to obesity and its expression might be related to its important role in the modulation of lipid metabolism. ${ }^{[33]}$

\section{CONCLUSION}

In the current study, it was proved that the concentrations of maternal circulating ZAG are significantly elevated in pregnancies complicated by PE. The results suggested that ZAG might have a role in the development of preeclampsia and could be served as a promising predictor marker for $\mathrm{PE}$ diagnosis.

\section{CONFLICT OF INTEREST}

There are no conflicts of interest

\section{REFERENCES}

1. Young BC, Levine RJ, Karumanchi SA. Pathogenesis of preeclampsia. Annu Rev Pathol 2010; 5:173-92. 
2. Stepan H, Philipp A, Roth I, Kralisch S, Jank A, Schaarschmidt W, et al. Serum levels of the adipokine zinc- $\alpha 2$-glycoprotein are increased in preeclampsia. J Endocrinol Invest 2012; 35(6):562-5.

3. Steegers EA, von Dadelszen P, Duvekot JJ, Pijnenborg R. Preeclampsia. Lancet 2010; 376:631-44.

4. Kharkova OA, Grjibovski AM, Krettek A, Nieboer E, Odland JO. First-trimester smoking cessation in pregnancy did not increase the risk of preeclampsia/ eclampsia: A Murmansk County Birth Registry study. PLoS One 2017;12(8):e0179354.

5. Stepan H, Geide A, Faber R. Soluble fms-like tyrosine kinase 1. N Engl J Med 2004; 351:22412.

6. Stepan H. Angiogenic factors and pre-eclampsia: an early marker is needed. Clin Sci (Lond) 2009; $116: 231-2$

7. Stepan H, Faber R. Elevated sFlt1 level and preeclampsia with parvovirus-induced hydrops. N Engl J Med 2006; 354:1857-8.

8. Stepan H, Unversucht A, Wessel N, Faber R. Predictive value of maternal angiogenic factors in second trimester pregnancies with abnormal uterine perfusion. Hypertension 2007; 49:818-24.

9. Bloomgarden ZT. Adiposity and diabetes. Diabetes Care 2002; 25:2342-9.

10. Kershaw EE, Flier JS. Adipose tissue as an endocrine organ. J Clin Endocrinol Metab 2004; 89:2548-56.

11. Arner P. Insulin resistance in type 2 diabetes -role of the adipokines. Curr Mol Med 2005; 5:333-9.

12. Fantuzzi G. Adipose tissue, adipokines, and inflammation. J Allergy Clin Immunol 2005; 115:911-9.

13. Bing C, Mracek T, Gao D, Trayhurn P. Zinc- $\alpha 2-$ glycoprotein: an adipokine modulator of body fat mass? Int J Obes (Lond) 2010; 34(11):1559-65

14. Mohamed-Ali V, Pinkney JH, Coppack SW. Adipose tissue as an endocrine and paracrine organ. Int J Obes Relat Metab Disord 1998; 22: 1145-58.

15. Ahima RS, Flier JS. Adipose tissue as an endocrine organ. Trends Endocrinol Metab 2000; 11: $327-32$
16. Fruhbeck G, Gomez-Ambrosi J, Muruzabal FJ, Burrell MA. The adipocyte: a model for integration of endocrine and metabolic signaling in energy metabolism regulation. Am J Physiol Endocrinol Metab 2001; 280: E827-47.

17. Scherer PE. Adipose tissue: from lipid storage compartment to endocrine organ. Diabetes 2006; 55:1537-45.

18. Zhu HJ, Wang XQ, Pan H, Gong FY, Zhang DX, Li NS, et al. Serum Levels of the Adipokine Zinc- $\alpha$ 2-glycoprotein Are Decreased in Patients with Hypertension. ISRN Endocrinol 2014; 2014:374090.

19. Zhou J, Qin G. Adipocyte dysfunction and hypertension. Am J Cardiovasc Dis 2012; 2:143-9.

20. Sanchez LM, Chirino AJ, Bjorkman PJ. Crystal structure of human ZAG, a fat-depleting factor related to MHC molecules. Science 1999; 283:1914-9.

21. Tada T, Ohkubo I, Niwa M, Sasaki M, Tateyama H, Eimoto T. Immunohistochemical localization of Zn-alpha 2- glycoprotein in normal human tissues. J Histochem Cytochem 1991; 39:1221-6.

22. Yang M, Liu R, Li S, Luo Y, Zhang Y, Zhang L, et al. Zinc- $\alpha 2$-glycoprotein is associated with insulin resistance in humans and is regulated by hyperglycemia, hyperinsulinemia, or liraglutide administration: cross-sectional and interventional studies in normal subjects, insulin-resistant subjects, and subjects with newly diagnosed diabetes. Diabetes Care 2013; 36(5):1074-82.

23. Marrades MP, Martínez JA, Moreno-Aliaga MJ. ZAG, a lipid mobilizing adipokine, is downregulated in human obesity. J Physiol Biochem 2008; 64:61-6.

24. Gong FY, Zhang SJ, Deng JY, Zhu HJ, Pan H, Li NS et al. Zinc-alpha2-glycoprotein is involved in regulation of body weight through inhibition of lipogenic enzymes in adipose tissue. Int $\mathbf{J}$ Obes (Lond) 2009; 33:1023-30.

25. Russell ST, Zimmerman TP, Domin BA, Tisdale MJ. Induction of lipolysis in vitro and loss of body fat in vivo by zinc-alpha2-glycoprotein. Biochim Biophys Acta 2004; 1636:59-68.

26. Price CP, Lamb EJ. Kidney Function Tests. In: Burtis CA, Ashwood ER, Bruns DE (eds). Tietz Textbook of Clinical Chemistry and Molecular Diagnostics. 5th ed. St. Louis: Saunders Elsevier 2012; 696-7. 
27. Okita K, Iwahashi H, Kozawa J, Okauchi Y, Funahashi T, Imagawa A, et al. Homeostasis model assessment of insulin resistance for evaluating insulin sensitivity in patients with type 2 diabetes on insulin therapy. Endocr J 2013; 60(3):283-90.

28. Craici IM, Wagner SJ, Bailey KR, Fitz-Gibbon PD, Wood-Wentz CM, Turner ST, et al. Podocyturia predates proteinuria and clinical features of preeclampsia: longitudinal prospective study. Hypertension 2013;61(6):1289-96.

29. Pelletier CC, Koppe L, Croze ML, Kalbacher E, Vella RE, Guebre-Egziabher F, et al. White adipose tissue overproduces the lipid-mobilizing factor zinc alpha2-glycoprotein in chronic kidney disease. Kidney Int 2013;83(5):878-86.

30. Philipp A, Kralisch S, Bachmann A, Lossner $\mathrm{U}$, Kratzsch J, Bluher $\mathrm{M}$, et al. Serum levels of the adipokine zinc-alpha2-glycoprotein are increased in chronic hemodialysis. Metabolism 2011;60(5):669-72.

31. Leal VO, Lobo JC, Stockler-Pinto MB, Farage NE, Abdalla DS, Leite M, Jr., et al. Is zinc-alpha2glycoprotein a cardiovascular protective factor for patients undergoing hemodialysis? Clin Chim Acta 2012;413(5-6):616-9.

32. Pelletier CC, Koppe L, Alix PM, Kalbacher E, Croze ML, Hadj-Aissa A, et al. The relationship between renal function and plasma concentration of the cachectic factor zinc-alpha2-glycoprotein (ZAG) in adult patients with chronic kidney disease. PLoS One 2014; 9(7):e103475.

33. Garrido-Sánchez L, García-Fuentes E, FernándezGarcía D, Escoté X, Alcaide J, Perez-Martinez P, et al. Zinc-Alpha 2-Glycoprotein Gene Expression in Adipose Tissue Is Related with Insulin Resistance and Lipolytic Genes in Morbidly Obese Patients. PLoS One 2012;7(3):e33264.

34. Tian M, Liang Z, Liu R, Li K, Tan X, Luo Y, et al. Effects of sitagliptin on circulating zincalpha2-glycoprotein levels in newly diagnosed type 2 diabetes patients: a randomized trial. Eur J Endocrinol 2016;174(2):147-55.

35. Xu L, Yu W, Niu M, Zheng C, Qu B, Li Y, et al. Serum ZAG Levels Were Associated with eGFR Mild Decrease in T2DM Patients with Diabetic Nephropathy. Int J Endocrinol 2017;2017:5372625.

36. Yeung DC, Lam KS, Wang Y, Tso AW, Xu A. Serum zinc-alpha2-glycoprotein correlates with adiposity, triglycerides, and the key components of the metabolic syndrome in Chinese subjects. J Clin Endocrinol Metab 2009;94(7):2531-6.

37. Stejskal D, Karpisek M, Reutova H, Stejskal P, Kotolova H, Kollar P. Determination of serum zinc-alpha-2-glycoprotein in patients with metabolic syndrome by a new ELISA. Clin Biochem 2008;41(4-5):313-6. 